\title{
Collapse-free action at a distance by multiple photon annihilations and tracking the action with a photon-number measurement
}

\author{
ShengLi Zhang 주 \\ Beijing Key Laboratory of Nanophotonics \& Ultrafine Optoelectronic Systems, School of Physics, \\ Beijing Institute of Technology, Beijing 100081, China
}

(Received 26 September 2019; revised 27 February 2020; accepted 11 May 2020; published 6 July 2020)

\begin{abstract}
The so-called quantum vampire effect, or, namely, collapse-free action at a distance by photon annihilation operator, is introduced in Optica 2, 112 (2015). Here, we investigate the quantum vampire effect in scenarios involving multiple photon annihilation on two different optical beams. We show that all $m$-time photon annihilations in some specific state will generate the same output, meaning that $m$ vampires can hide both their locations and their numbers in each optical mode. This demonstrates another interesting and strange phenomenon of quantum optics that originates from the property of the annihilation operator: $\hat{a}|0\rangle=0$. However, the annihilation operator is a nonphysical and nonunitary operation, and realistic implementation of photon annihilation is performed with the beam splitter and photon detector. We find that realistic photon annihilation will "leave" some shadow that makes the quantum vampire easier to track in real scenarios. We design a simple quantum measurement to catch realistic quantum vampires.
\end{abstract}

DOI: 10.1103/PhysRevA.102.013705

\section{INTRODUCTION}

The counterintuitive nature of quantum physics has led to many brilliantly innovative breakthroughs in information processing, such as quantum cryptography [1], quantum metrology [2], quantum teleportation [3], and quantum computing [4]. Recently, another counterintuitive effect known as the quantum vampire effect was discovered by a group of scientists from Russia and Canada [5]. The idea of a quantum vampire is that if some single mode state of light, for example, the Fock state $|n\rangle$, is distributed between two modes, and photon subtraction takes place in a certain mode, it changes the state in all the modes and it is equivalent to photon subtraction in the initial mode. Later, it was shown that such a phenomenon also occurs if the Fock state is replaced with a classic single mode thermal state [6]. The authors show, both theoretically and experimentally, that photon annihilation taking place in one mode of a beam-split thermal state leads to photon annihilation in the remaining modes as well. The basis behind quantum vampirism is that photon subtraction does not change the spatial intensity distribution [5,7] and thus does not cast a shadow. Such a property has intrinsically important relevance to eavesdropping with beam splitting [8-10] in quantum key distribution, as well as for developing quantum cloaking devices [5]. Recently, a direct test for the absence of

Published by the American Physical Society under the terms of the Creative Commons Attribution 4.0 International license. Further distribution of this work must maintain attribution to the author(s) and the published article's title, journal citation, and DOI. shadow has been experimentally demonstrated with a beam in the thermal state through multimode detection [7].

The quantum vampire effect has attracted significant research interest. The study of $k$-photon subtraction on a single mode has been investigated in Ref. [6] and it is suggested that a quantum vampire can be also transferred to $\hat{a}^{\dagger}$, the photon creation process [7]. In this paper, we extend the study of the quantum vampire effect to the case of $m$ times photon annihilation operations involving two optical modes ( $k$-time annihilation on the first mode and $(m-k)$-time annihilation on a second mode). We show that, for ideal photon annihilation, there exists a state $\left|\psi_{A B}\right\rangle$ for which all configurations of $m$ times of photon annihilations performed on the twomode state $\left|\psi_{A B}\right\rangle$ will generate the same normalized output state. This means that, for $\left|\psi_{A B}\right\rangle$, single photon annihilation performed on either mode yields the same output, and that it is impossible to identify from just the output state the mode that has been subjected to a photon annihilation operation. Thus no shadow is cast if we perform the annihilation operation on a single mode of $\left|\psi_{A B}\right\rangle$. Our result can be considered as a further extension of the effect of the original single quantum vampire [5] to a group of $m$ quantum vampires.

As an ideal annihilation operation is nonphysical and nonunitary, it is of interest to investigate whether it is possible to catch a quantum vampire. We find that the answer is affirmative. The realistic photon annihilation operation, implemented with an optical beam splitter and photon detector, does leave trace of its presence. We design a realistic measurement scheme with mode-by-mode photon-number detection to catch the quantum vampires.

This research study is organized as follows. Initially, in Sec. II A, we demonstrate the absence of the quantum vampire's shadow in the case of a one-time photon annihilation. 
In Sec. II B, we generalize our result to an arbitrary number of photon annihilations and show that all the different configurations of $m$-time photon annihilation will generate the same outputs. Section III is devoted to the investigation of the vampire's absence of shadow in the presence of realistic photon annihilation with a beam splitter and photon detectors. We use the minimal probability of erroneous inference to evaluate the distinguishability of the output state in different configurations of photon annihilation operations. In Sec. IV, we investigate the possibility of catching quantum vampires with quantum measurement. We design a mode-by-mode measurement to catch the vampires. Finally, our conclusion follows in Sec. V.

\section{SCHEME OF A QUANTUM VAMPIRE AT IDEAL PHOTON ANNIHILATION}

\section{A. One-time photon annihilation}

The explanation of the quantum vampire phenomenon is that shadows are caused by the absorption of light. From the governing equation [11], the state evolution follows: $d \rho / d t=$ $\hat{a} \rho \hat{a}^{\dagger}-\left(\rho \hat{a} \hat{a}^{\dagger}+\hat{a} \hat{a}^{\dagger} \rho\right) / 2$. However, the photon annihilation is a manipulation of the photon number that will never change the shape profile [7] of the input state. A scheme is shown in Figs. 1(a1) and 1(a2) to show the difference between linear absorption and photon annihilation.

Figures 1(b1) and 1(b2) shows a stronger property of the quantum vampire's shadow absence in the two-mode system. Let the annihilation operation denote the presence of the quantum vampire. It may present itself in mode $A$ or $B$ (window $A$ or $B$ ). It can be easily demonstrated that the photon annihilation on mode $A$ or $B$ will generate the same output state. Such a conclusion holds for all Fock states $|n\rangle(n \geqslant 0)$ and all beam splitters $T(0<T<1)$. This implies that the location of the quantum vampire can never be traced, if only the output state alone can be accessed.

Let the input state of optical mode $B_{0}$ be a vacuum. The coupling between $A_{0}$ and $B_{0}$, via the beam splitter with transmittance $T$, will generate an entangled state:

$$
\begin{aligned}
\left|\psi_{A B}\right\rangle & =U\left(|n\rangle_{A_{0}}|0\rangle_{B_{0}}\right) \\
& =\exp \left[\arctan \left(a_{A} a_{B}^{\dagger}-a_{A}^{\dagger} a_{B}\right)\right]|n\rangle_{A_{0}}|0\rangle_{B_{0}} \\
& =\sum_{k=0}^{n} \sqrt{\left(\begin{array}{l}
n \\
k
\end{array}\right)}(T)^{\frac{n-k}{2}}(1-T)^{\frac{k}{2}}|n-k\rangle_{A}|k\rangle_{B} .
\end{aligned}
$$

Next, we consider the output state after the one-time photon annihilation (unnormalized):

$$
\begin{aligned}
\left|\widetilde{\psi}_{A B}^{(1,0)}\right\rangle & =\left(\hat{a}_{A} \otimes I\right)\left|\psi_{A B}\right\rangle \\
& =\sum_{k=0}^{n-1} \sqrt{\left(\begin{array}{l}
n \\
k
\end{array}\right)}(T)^{\frac{n-k}{2}}(1-T)^{\frac{k}{2}} \sqrt{n-k}|n-k-1\rangle|k\rangle,
\end{aligned}
$$

$$
\begin{aligned}
\left|\widetilde{\psi}_{A B}^{(0,1)}\right\rangle & =\left(I \otimes \hat{a}_{A}\right)\left|\psi_{A B}\right\rangle \\
& =\sum_{k=1}^{n} \sqrt{\left(\begin{array}{l}
n \\
k
\end{array}\right)}(T)^{\frac{n-k}{2}}(1-T)^{\frac{k}{2}} \sqrt{k}|n-k\rangle|k-1\rangle .
\end{aligned}
$$
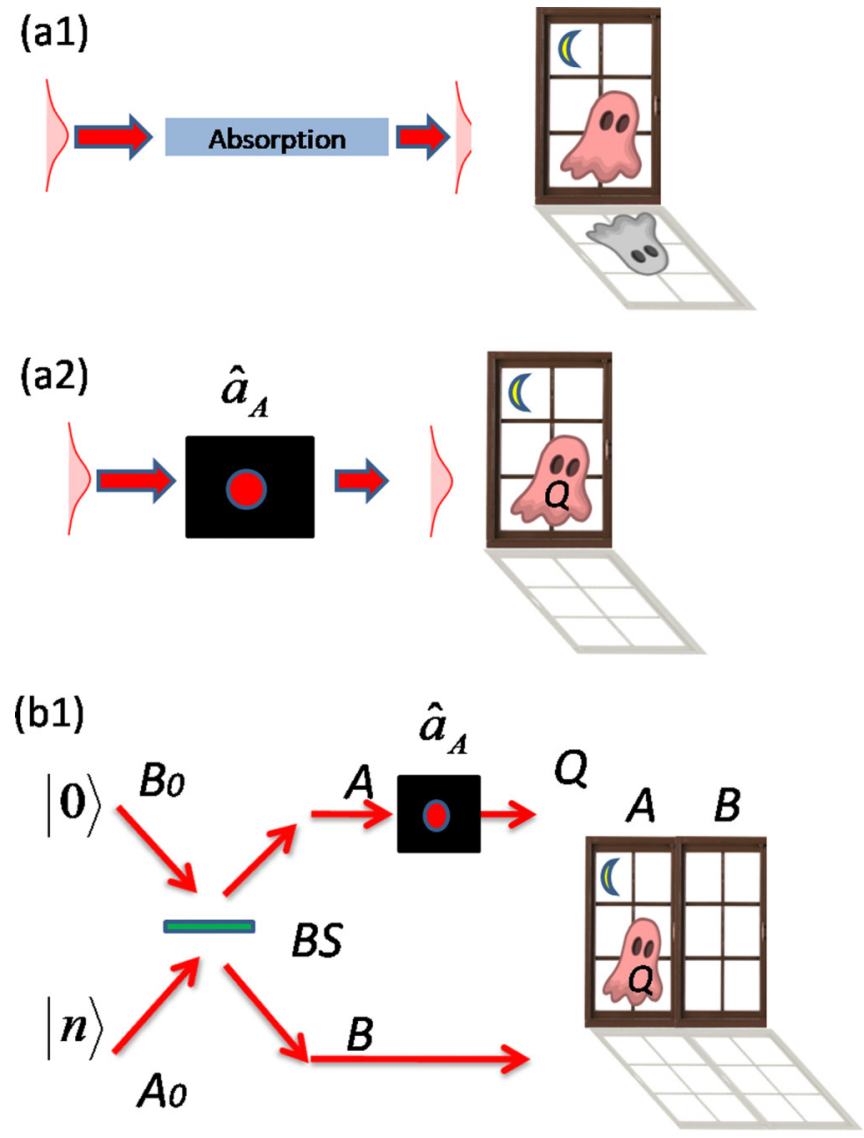

\section{(b2)}

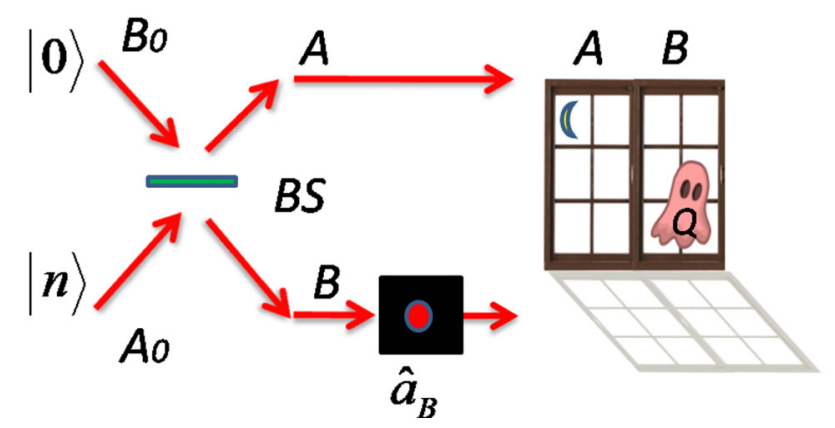

FIG. 1. (a1) Shadow caused by linear absorption of light. (a2) The absence of the quantum vampire's shadow represented by the photon annihilation operator. (b1) One-time photon annihilation on mode $A$. (b2) One-time photon annihilation on mode $B$. All states in mode $A_{0}$ of (b1) and (b2) are initialized with Fock state $|n\rangle$. The transmittance of all beam splitters (BS) are set to $T$ for simplicity.

After some straightforward calculations, we find that

$$
\left|\widetilde{\psi}_{A B}^{(1,0)}\right\rangle=\sqrt{\frac{T}{1-T}}\left|\widetilde{\psi}_{A B}^{(0,1)}\right\rangle .
$$

In the following, we will use the symbol $\propto$ to show such an equality relation if the normalization constant is neglected:

$$
\left|\widetilde{\psi}_{A B}^{(1,0)}\right\rangle \propto\left|\widetilde{\psi}_{A B}^{(0,1)}\right\rangle
$$

Thus the two schemes of one-time photon annihilation generate the same output state (up to an unobservable normalization 


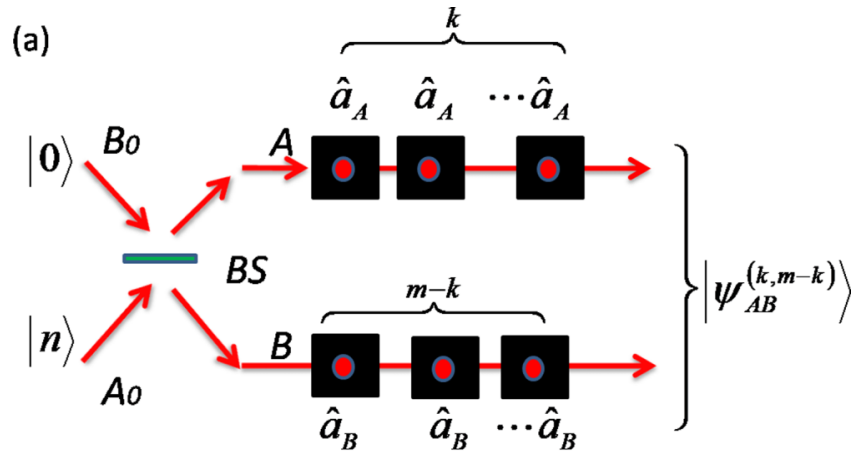

(b)

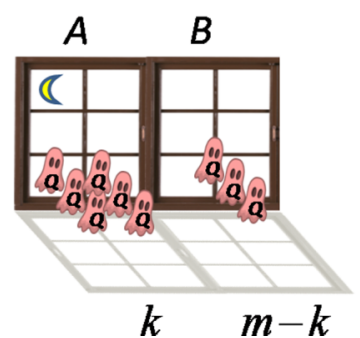

FIG. 2. (a) Scheme of $m$ photon subtraction on beam-split Fock state. (b) Illustration of $k$ quantum vampires in window $A$ and $m-k$ quantum vampires in windows $B$. The transmittance of the beam splitters (BS) are set to $T$ for simplicity.

constant). No information about the location of the quantum vampire is saved in the outputs.

\section{B. Case of a quantum vampire with multiple photon annihilations}

It is interesting to investigate the property of multiple photon annihilations (see Fig. 2). Let the unnormalized state $\left|\widetilde{\psi}^{(k, m-k)}\right\rangle=\hat{a}_{A}^{\otimes k} \otimes \hat{a}_{B}^{\otimes m-k}\left|\psi_{A B}\right\rangle$ denote the $m$ times of photon annihilation operations [ $k$-time annihilation on mode $A$ and $(m-k)$-time annihilation on mode $B]$. It can be shown that all configurations of $m$ times of photon annihilations performed on the two-mode state $\left|\psi_{A B}\right\rangle$ will generate the same normalized output state. For $m=1$ we have only two kinds of one-time photon annihilations: $(1,0)$ and $(0,1)$. The proof is given in Eq. (4).

For $m=2$, we have three kinds of configuration: $(2,0)$, $(1,1)$, and $(0,2)$. Applying single photon annihilation on $A$ mode of $\left|\widetilde{\psi}_{A B}^{(1,0)}\right\rangle$ and $\left|\widetilde{\psi}_{A B}^{(0,1)}\right\rangle$, it follows [taking account of Eq. (4)] that

$$
\begin{aligned}
\left|\widetilde{\psi}_{A B}^{(2,0)}\right\rangle & \equiv\left(\hat{a}_{A} \otimes I\right)\left|\widetilde{\psi}_{A B}^{(1,0)}\right\rangle \\
& =\sqrt{\frac{T}{1-T}}\left(\hat{a}_{A} \otimes I\right)\left|\widetilde{\psi}_{A B}^{(0,1)}\right\rangle \propto\left|\widetilde{\psi}_{A B}^{(1,1)}\right\rangle .
\end{aligned}
$$

Applying single photon annihilation on $B$ mode, we obtain

$$
\left|\widetilde{\psi}_{A B}^{(1,1)}\right\rangle \propto\left|\widetilde{\psi}_{A B}^{(0,2)}\right\rangle .
$$

Combining Eqs. (7) and (8), we conclude that all three configurations of the two-time photon annihilation generate exactly the same output pure state (up to a normalization factor).
This proof can be generalized in a straightforward manner via mathematical induction to arbitrary $m$-time photon annihilation.

\section{REALISTIC PHOTON ANNIHILATION WITH BEAM SPLITTER AND PHOTON DETECTORS}

In the analysis above, we have considered the ideal photon annihilation operation. In reality, photon annihilation operations are a nonphysical operation and a real life photon annihilation operation can be implemented with a photon beam splitter and photon detectors [12]. Such a method has been widely used in continuous-variable entanglement distillation [13-19] and test of commutation relation [20,21].

Ideal quantum vampires with photon annihilation can hide their locations (Sec. II) and their numbers (Sec. II B). However, practical quantum vampires with realistic photon subtraction can be detected. We consider the one-time realistic photon subtraction as an example. Again, we regard the Fock state as input. Realistic photon subtraction is always implemented by coupling the relevant optical mode with a vacuum ancilla. Subsequent detection of photons in the outgoing ancillary mode will herald a successful photon subtraction. A practical scheme is depicted in Fig. 3.

It should be noted that the photon subtraction in this case approximates the ideal photon annihilation very well when we apply a highly transparent beam splitter, and when the input state is mainly populated in the weakly excited Fock state ( $n$ small) [22].

In the Fock state basis, it follows that realistic photon annihilation on modes $A$ and $B$ will generate (normalized, see Appendix A for more information)

$$
\begin{gathered}
\left\langle 00\left|\rho^{(1,0)}\right| 00\right\rangle=\frac{T^{n}\left(1-T^{\prime}\right)^{n}}{1-\left(1-T+T T^{\prime}\right)^{n}}, \\
\left\langle 00\left|\rho^{(0,1)}\right| 00\right\rangle=\frac{(1-T)^{n}\left(1-T^{\prime}\right)^{n}}{1-\left(T+T^{\prime}-T T^{\prime}\right)^{n}} .
\end{gathered}
$$

After direct calculation, we find that the output states that depend on a successful photon subtraction are mixed states. Detailed analysis shows that the fidelity with respect to ideal photon annihilation will improve if a beam splitter with a higher transmittance is used (see Appendix B).

Thus $\rho^{(1,0)}$ and $\rho^{(0,1)}$ are two distinct states. We can use optimal quantum measurements to carry out a minimalerror-probability discrimination of $\rho^{(1,0)}$ and $\rho^{(0,1)}$ [23-25]. The minimal error probability for the discrimination follows $P_{\text {err }}=\frac{1}{2}\left(1-\frac{1}{2}\left\|\rho^{(1,0)}-\rho^{(0,1)}\right\|\right)$ when the optimal joint measurement induced by the Schur transformation is performed $[26,27]$.

In the following, we use $P_{\text {err }}$ as a figure of merit to evaluate the distinguishability of the output states generated in the various configurations of photon annihilations. An error probability of $P_{\text {err }}=1 / 2$ corresponds to the case when two cases of realistic photon subtractions yield the same outputs.

$P_{\text {err }}=0$ corresponds to the perfect discrimination of two orthogonal states. Figure 4 shows the error probability of optimal discrimination of $\rho^{(1,0)}$ and $\rho^{(0,1)}$, for a varying transmittance $T^{\prime}$ used for photon subtraction. All Fock states are chosen as $n=5$ and all states of the relevant optical modes 
(a)

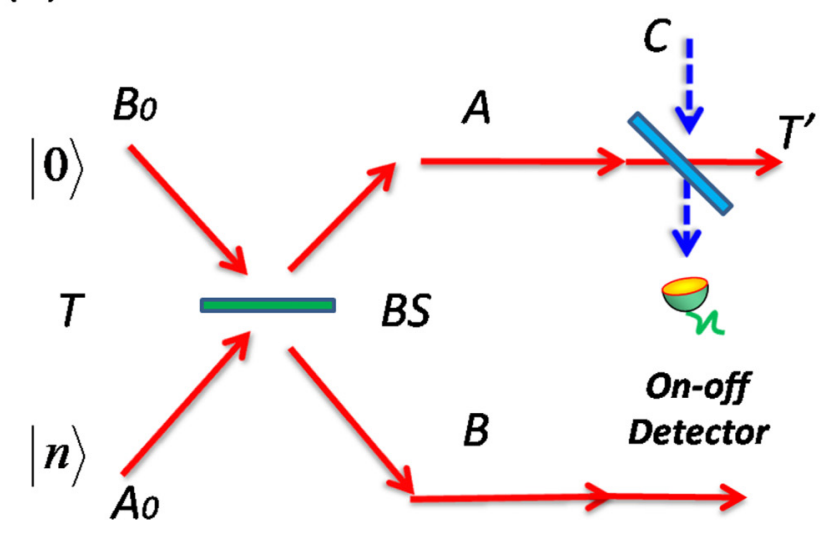

(b)

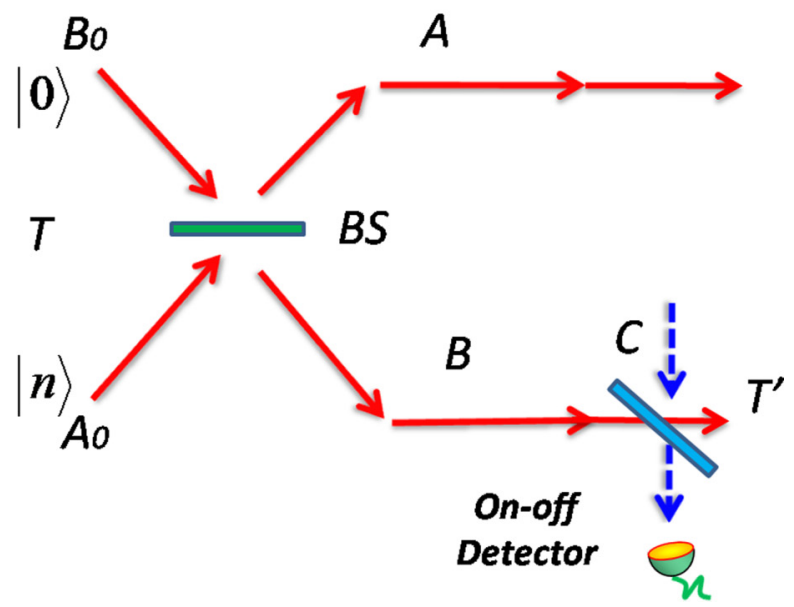

FIG. 3. Realistic one-time photon annihilation with optical beam splitter (with transmittance $T^{\prime}$ ) and photon detectors. (a) One-time photon annihilation on mode $A$. (b) One-time photon annihilation on mode $B$. Successful photon annihilation is heralded when the on-off photon detector registers "on" results.

are truncated within the subspace spanned by $\{|0\rangle,|1\rangle,|2\rangle$, $|D-1\rangle\}$ and $D=6$ if they are not specified. It is evident that it is more difficult to discriminate the output state generated by photon subtraction with a higher $T^{\prime}$. This agrees with the fidelity analysis presented in Appendix B.

It is also quite interesting to show the distinguishability of two-time realistic photon subtraction. According to Sec. II B, there are three different allocations of two-time photon subtraction on the two modes $A$ and $B:(2,0),(1,1)$, and $(0,2)$. The distinguishability of these three cases can be obtained by the minimal error discrimination of the corresponding sets of states $\left\{\rho^{(2,0)}, \rho^{(1,1)}\right\},\left\{\rho^{(1,1)}, \rho^{(0,2)}\right\}$, and $\left\{\rho^{(2,0)}, \rho^{(0,2)}\right\}$. The corresponding error probability is represented by $P_{\mathrm{err}}^{(1)}, P_{\mathrm{err}}^{(2)}$, and $P_{\mathrm{err}}^{(3)}$ in Fig. 5.

In the following, we investigate the question of whether the quantum vampire can be made totally indiscriminate if the apparatus used is equipped with a photon-number-resolving

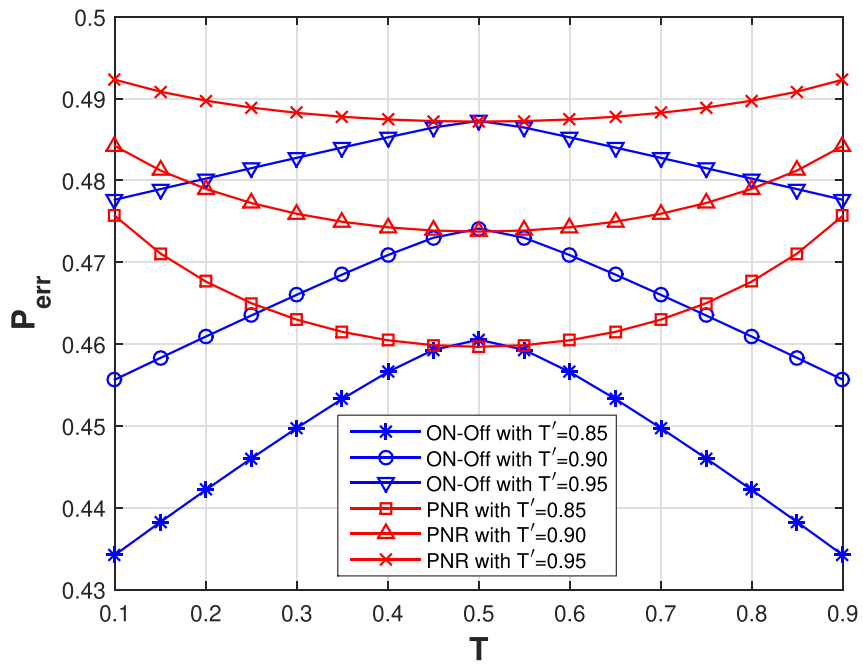

FIG. 4. Minimal error probability as a function of varying transmittance $T$ for one-time photon annihilation.

(PNR) detector. Unfortunately, the answer is still negative. With the development of superconducting techniques, photon detectors with the ability to perform photon-number resolution are available in state-of-art laboratories [28,29]. We find that the output state conditioned upon a real single photon detected in the PNR detector is still a pure state. Written in Fock state space, it follows (normalized) that

$$
\begin{aligned}
\left|\psi_{\mathrm{PNR}}^{(1,0)}\right\rangle= & \frac{1}{\sqrt{N_{\mathrm{PNR}}^{(1,0)}}}{ }_{C}\left\langle 1\left|U_{A C}\left(T^{\prime}\right) U_{A_{0} B_{0}}(T)\right| n\right\rangle_{A}|0\rangle_{B}|0\rangle_{C} \\
= & \left(1-T+T T^{\prime}\right)^{-\frac{n-1}{2}} \sum_{k=0}^{n-1} \sqrt{\left(\begin{array}{c}
n-1 \\
k
\end{array}\right)} \\
& \times\left(T T^{\prime}\right)^{\frac{n-1-k}{2}}(1-T)^{\frac{k}{2}}|n-1-k\rangle_{A}|k\rangle_{B}, \quad(11) \\
\left|\psi_{\mathrm{PNR}}^{(0,1)}\right\rangle= & \frac{1}{\sqrt{N_{\mathrm{PNR}}^{(0,1)}} C\left\langle 1\left|U_{B C}\left(T^{\prime}\right) U_{A_{0} B_{0}}(T)\right| n\right\rangle_{A}|0\rangle_{B}|0\rangle_{C}} \\
= & \left(T+T^{\prime}-T T^{\prime}\right)^{-\frac{n-1}{2}} \\
& \times \sum_{k=0}^{n-1} \sqrt{\left(\begin{array}{c}
n-1 \\
k
\end{array}\right)} T^{\frac{n-k-1}{2}}\left[(1-T) T^{\prime}\right]^{\frac{k}{2}}|n-k-1\rangle|k\rangle .
\end{aligned}
$$

The conditioned state using the PNR detector is pure, which is very different from the case for the mixed state [Eqs. (A3) and (A6)] when using an on-off photon detector. This is because, in the case of a PNR detector, the ancillary $C$ mode is projected to $|1\rangle\langle 1|$, whereas, in the case of an on-off detector, all the projections of the ancillary $C$ mode to $|1\rangle\langle 1|| 2\rangle,\langle 2|| 3\rangle,\langle 3| \ldots$ are considered. In actuality, only the projection to $|1\rangle\langle 1|$ represents the ideal single photon subtraction case; thus the scheme using the PNR detector scheme will have the higher fidelity with respect to ideal photon subtraction. 


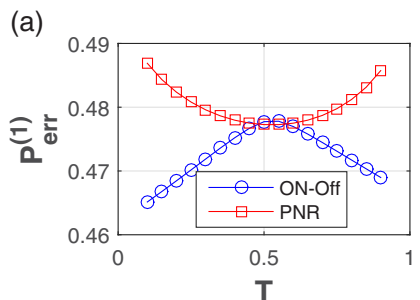

(b)
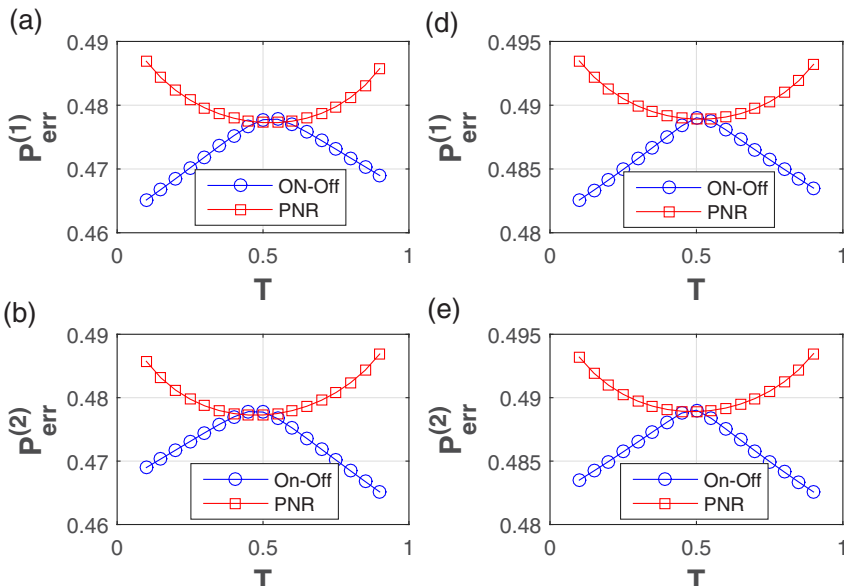

(c)

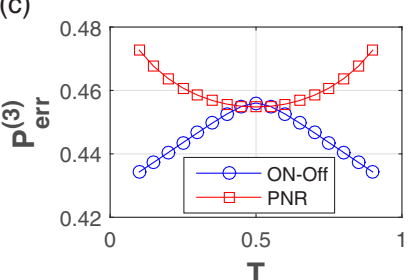

(e)

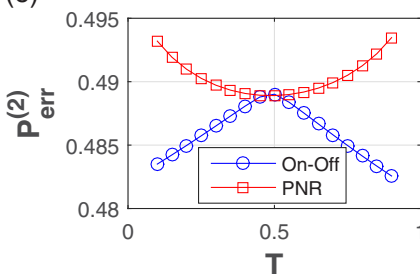

(f)

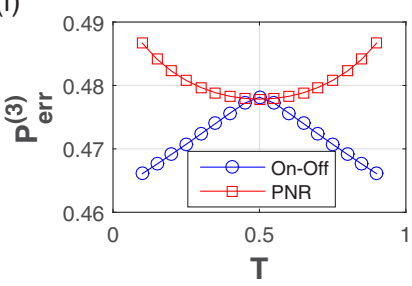

FIG. 5. Minimal error probability as function of varying transmittance $T$ for two-time photon annihilation. Other parameters: (a), (b), (c) $T^{\prime}=0.90$; (d), (e), (f) $T^{\prime}=0.95$.

However, it is easily verified that

$$
\begin{aligned}
\left\langle n-1,0 \mid \psi_{\mathrm{PNR}}^{(1,0)}\right\rangle & =\left(\frac{T T^{\prime}}{1-T+T T^{\prime}}\right)^{\frac{n-1}{2}} \\
\neq\left\langle n-1,0 \mid \psi_{\mathrm{PNR}}^{(0,1)}\right\rangle & =\left(\frac{T}{T+T^{\prime}-T T^{\prime}}\right)^{\frac{n-1}{2}} .
\end{aligned}
$$

Thus photon subtraction with a PNR detector cannot still replace the ideal photon annihilation in the test of the quantum vampire. However, it can be easily observed that the error probability is much larger, as shown by the lines marked with red squares in Figs. 4 and 5. This means that photon subtraction with PNR has more fidelity with the ideal photon annihilation and it is more difficult to discriminate the output state.

\section{CATCHING A QUANTUM VAMPIRE WITH REALISTIC PHOTON-NUMBER MEASUREMENTS}

As a realistic quantum vampire implemented with a realistic beam splitter and photon detector will always leave some trace, it is of interest to demonstrate how to observe its presence with physical feasible measurement. In Sec. III, it is shown that the error probability of identifying a single vampire and two vampires is about 0.47 , which is relatively high considering that a random guess would give an error probability of 0.50 . Thus, to further decrease the error probability, one could use a multicopy version. Suppose that one or two quantum vampires appear in the same location many times. Then, one can use quantum measurement, such as photon-number measurement, to capture its (their) presence. This is quite analogous to fictional stories of vampires: a

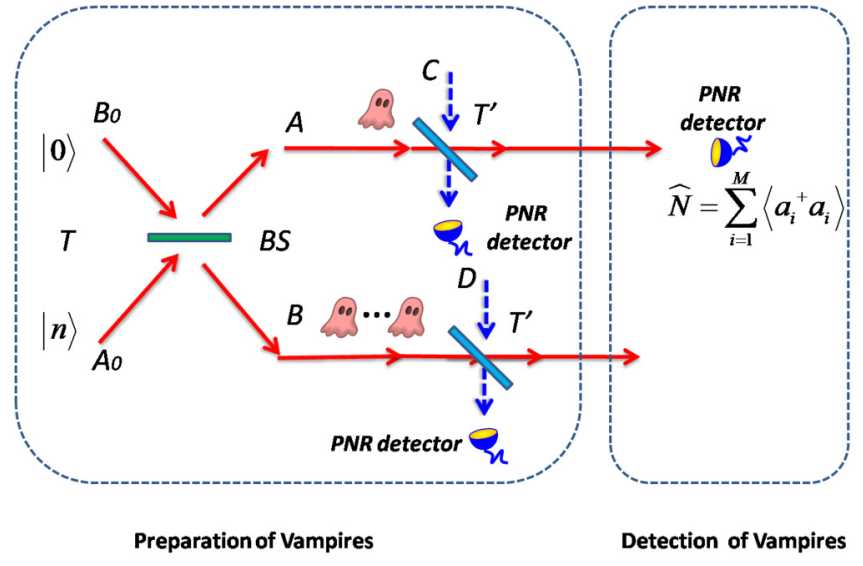

FIG. 6. Scheme of preparation and detection of quantum vampires. A photon-number-resolving detector is applied and $M$-copy states are used to further decrease the error probability.

single appearance may be easily neglected or it may even be considered as a normal being; only multiple appearances of a vampire could incur adequate attention. We divide the following discussions into two subsections: one quantum vampire and two quantum vampires.

\section{A. One quantum vampire}

For convenience, we consider the implementation of a quantum vampire with PNR photon detectors. The task is then to discriminate $\left|\psi_{\mathrm{PNR}}^{(1,0)}\right\rangle$ [generated by Fig. 3(a)] with the detector replaced by a PNR detector, and $\left|\psi_{\mathrm{PNR}}^{(0,1)}\right\rangle$ [generated by Fig. 3(b) with the detector replaced by a PNR detector]. Moreover, we have a multicopy at hand: $\left|\psi_{\mathrm{PNR}}^{(1,0)}\right\rangle^{\otimes M}$ and $\left|\psi_{\mathrm{PNR}}^{(0,1)}\right\rangle^{\otimes M}$. For discrimination of the $M$-copy state, one could use the quantum Chernoff bound (QCB) [26,27] to evaluate the minimal error probability. The quantum Chernoff bound is achieved with joint measurement on the $M$-copy mixed state, which requires a large-scale quantum memory to all the $M$-copy quantum states. A more practical method may be mode-by-mode or copy-by-copy local measurements.

A scheme of catching a single vampire is shown in Fig. 6. We use a photon-number-resolving detector to detect the photon in the optical $A$ mode. If the vampire appears in $A$ mode, it follows that the mean and variance of photon number in $A$ mode is

$$
\begin{aligned}
\bar{n} & =\left\langle\psi_{\mathrm{PNR}}^{(1,0)}\left|a_{A}^{\dagger} a_{A} \otimes I\right| \psi_{\mathrm{PNR}}^{(1,0)}\right\rangle \\
& =\frac{(n-1) T T^{\prime}}{1-T+T T^{\prime}}, \\
V & =\left\langle\psi_{\mathrm{PNR}}^{(1,0)}\left|\left(a_{A}^{\dagger} a_{A}\right)^{2} \otimes I\right| \psi_{\mathrm{PNR}}^{(1,0)}\right\rangle-\bar{n}^{2} \\
& =\frac{(n-1)(1-T) T T^{\prime}}{\left(1-T+T T^{\prime}\right)^{2}} .
\end{aligned}
$$

However, if the vampire appears in $B$ mode, it follows that

$$
\begin{aligned}
\bar{n}^{\prime} & =\left\langle\psi_{\mathrm{PNR}}^{(0,1)}\left|a_{A}^{\dagger} a_{A} \otimes I\right| \psi_{\mathrm{PNR}}^{(0,1)}\right\rangle \\
& =\frac{(n-1) T}{T+T^{\prime}-T T^{\prime}},
\end{aligned}
$$




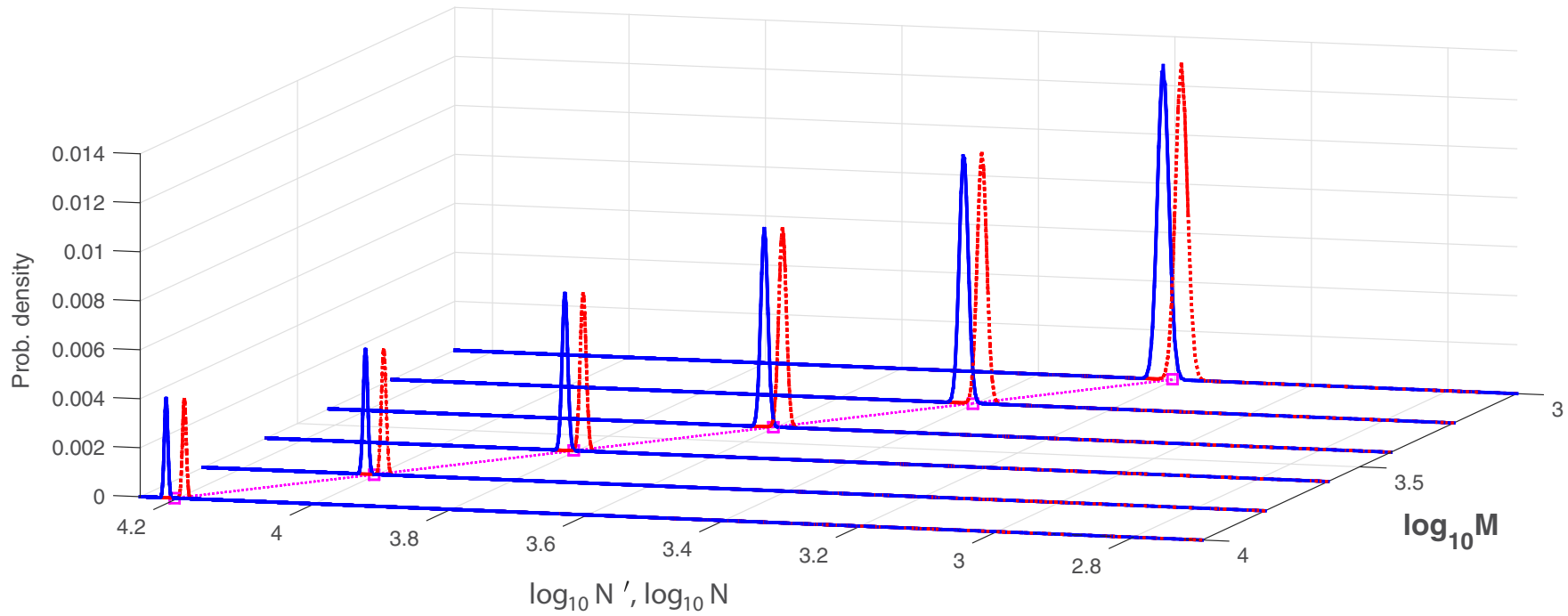

FIG. 7. Probability density of Gaussian distribution $\mathcal{N}$ (dotted red line, in logarithmic scale) and $\mathcal{N}^{\prime}$ (solid blue line, in logarithmic scale) for increasing values of $M$. Other parameters are as follows: $T=0.40, T^{\prime}=0.95, D=6, n=5$. Lines marked with squares indicate the $N_{\text {th }}$ value of the corresponding $M$.

$$
\begin{aligned}
V^{\prime} & =\left\langle\psi_{\mathrm{PNR}}^{(0,1)}\left|\left(a_{A}^{\dagger} a_{A}\right)^{2} \otimes I\right| \psi_{\mathrm{PNR}}^{(0,1)}\right\rangle-\bar{n}^{\prime 2} \\
& =\frac{(n-1)(1-T) T T^{\prime}}{\left(T+T^{\prime}-T T^{\prime}\right)^{2}} .
\end{aligned}
$$

This is the simplest case of $M=1$. For the $M$-copy version, it follows that the summation of all $M$ instances of detection results follows a Gaussian distribution (for sufficiently $\operatorname{large} M)$ :

$$
\begin{aligned}
N & =\sum_{i=1}^{M} n_{i} \sim \mathcal{N}(M \bar{n}, M V), \\
N^{\prime} & =\sum_{i=1}^{M} n_{i}^{\prime} \sim \mathcal{N}^{\prime}\left(M \bar{n}^{\prime}, M V^{\prime}\right),
\end{aligned}
$$

with $n_{i}\left(n_{i}^{\prime}\right)$ being the number of detected photons in the $i$ th photon-number measurement if the state $\left|\psi_{\mathrm{PNR}}^{(1,0)}\right\rangle\left(\left|\psi_{\mathrm{PNR}}^{(0,1)}\right\rangle\right)$ is at hand and $\mathcal{N}, \mathcal{N}^{\prime}$ being two Gaussian distributions.

As $0<T, T^{\prime}<1$, it is easily obtained that $\bar{n}<\bar{n}^{\prime}$. The task of discriminating the location of the vampire in optical modes $A$ and $B$ is equivalent to the discrimination of the two Gaussian distributions of Eqs. (21) and (22).

Let an estimator $\hat{N}$ be the summation of all $M$ results for photon-number detection. We can establish a rule to infer the location of the vampire:

$$
\begin{aligned}
& A \Leftrightarrow\left|\psi_{\mathrm{PNR}}^{(1,0)}\right\rangle \Leftrightarrow \hat{N}<N_{\mathrm{th}}, \\
& B \Leftrightarrow\left|\psi_{\mathrm{PNR}}^{(0,1)}\right\rangle \Leftrightarrow \hat{N} \geqslant N_{\mathrm{th}} .
\end{aligned}
$$

As we have no a priori information on the presence of the vampire, we can assume that it will appear in mode $A$ or mode $B$, both with probability $1 / 2$. The error of our discrimination is given by

$$
P_{\mathrm{err}}=\frac{1}{2} P\left(\hat{N}>N_{\mathrm{th}} \mid \mathcal{N}\right)+\frac{1}{2} P\left(\hat{N}<N_{\mathrm{th}} \mid \mathcal{N}^{\prime}\right) .
$$

For convenience, one can define $N_{\text {th }}$ such that two kinds of error are equal: $P\left(\hat{N}>N_{\text {th }} \mid \mathcal{N}\right)=P\left(\hat{N}<N_{\text {th }} \mid \mathcal{N}^{\prime}\right)$. According to Gaussian distribution, it follows after straightforward calculation that

$$
N_{\text {th }}=\frac{M\left(\bar{n}^{\prime} \sqrt{V}+\bar{n} \sqrt{V^{\prime}}\right)}{\sqrt{V}+\sqrt{V^{\prime}}}
$$

and the probability of erroneous inference of the vampire's location in the case of $M$-copy inputs is given by

$$
P_{\text {err }}=\frac{1}{2} \operatorname{erfc}\left(\sqrt{\frac{M}{2}} \frac{\left|\bar{n}-\bar{n}^{\prime}\right|}{\sqrt{V}+\sqrt{V^{\prime}}}\right) .
$$

Using the approximation $\operatorname{erfc}(y)=e^{-y^{2}} /(\sqrt{\pi} y), \quad y \gg 1$, an exponentially decreasing error probability can be obtained:

$$
\begin{gathered}
P_{\mathrm{err}} \approx \frac{e^{-M R_{\mathrm{eff}}}}{2 \sqrt{\pi M R_{\mathrm{eff}}}}, \\
R_{\mathrm{eff}}=\frac{\left(\bar{n}-\bar{n}^{\prime}\right)^{2}}{2\left(\sqrt{V}+\sqrt{V^{\prime}}\right)^{2}} .
\end{gathered}
$$

Thus one sees that the identification of the quantum vampire is successful with the error probability decreasing towards zero exponentially.

For example, we can give a numerical dependence of statistics $\mathcal{N}$ and $\mathcal{N}^{\prime}$ in Fig. 7. We consider the case $T=$ $0.40, T^{\prime}=0.95, n=5$. It can be seen that the two peaks of $\mathcal{N}$ and $\mathcal{N}^{\prime}$ are separated greatly by increasing the value of copy number $M$. For $M=10^{3.2}=1584$, the two Gaussian distributions of $\mathcal{N}$ and $\mathcal{N}^{\prime}$ are separated.

The explicit probability $P_{\text {err }}$ [Eq. (26)] is plotted with circles in Fig. 8(a). A line marked with asterisks indicates the quantum Chernoff bound of optimal discrimination of $M$-copy $\left|\psi_{\mathrm{PNR}}^{(1,0)}\right\rangle$ and $\left|\psi_{\mathrm{PNR}}^{(0,1)}\right\rangle$ :

$$
\begin{gathered}
P_{\mathrm{QCB}}=\frac{1}{2} Q^{M}, \\
Q=\min _{0 \leqslant s \leqslant 1} \operatorname{Tr}\left[\rho_{0}^{s} \rho_{1}^{1-s}\right], \\
\rho_{0}=\left|\psi_{\mathrm{PNR}}^{(1,0)}\right\rangle\left\langle\psi_{\mathrm{PNR}}^{(1,0)}\left|, \quad \rho_{1}=\right| \psi_{\mathrm{PNR}}^{(0,1)}\right\rangle\left\langle\psi_{\mathrm{PNR}}^{(0,1)}\right| .
\end{gathered}
$$


(a)

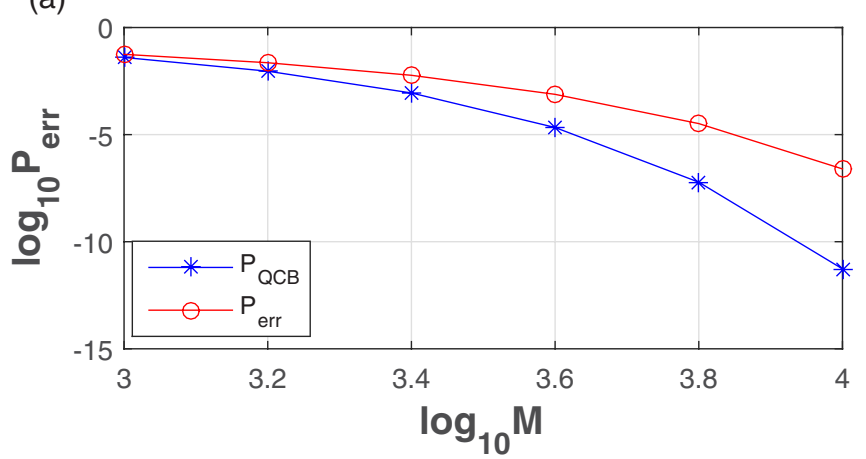

(b)

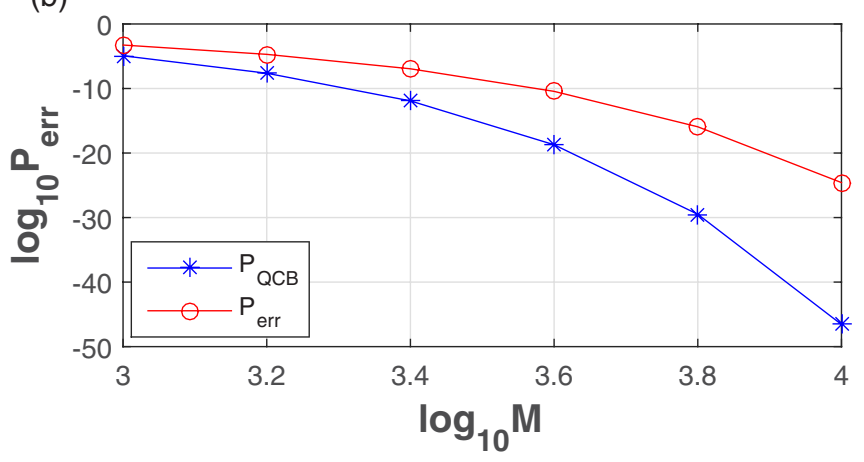

FIG. 8. Quantum Chernoff bound ( $P_{\mathrm{QCB}}$, in logarithmic scale) and error probability $\left[P_{\mathrm{err}}\right.$, Eq. (26), in logarithmic scale] for $T=0.40, D=6, n=5$. Other parameters are (a) $T^{\prime}=0.95$ and (b) $T^{\prime}=0.90$.

For example, for $M=10^{3.2} \approx 1584$, the error probability of mode-by-mode measurement is $P_{\text {err }}=0.0226$ and the $\mathrm{QCB}$ bound is $P_{\mathrm{QCB}}=0.0091$. Although our mode-by-mode scheme has a higher probability than $P_{\mathrm{QCB}}, P_{\text {err }} \ll 0.47$ is sufficient to prove that the location of a vampire can be identified with a high probability of success.

In Fig. 8(b), we demonstrate an evaluation of the discrimination of $M$-copy $\left|\psi_{\mathrm{PNR}}^{(1,0)}\right\rangle$ and $\left|\psi_{\mathrm{PNR}}^{(0,1)}\right\rangle$ generated by the beam splitter with $T^{\prime}=0.90$. Comparing it with the case when $T^{\prime}=0.95$, the transmittance is lower and the fidelity with respect to ideal photon subtraction is lower. The corresponding state is easier to discriminate and thus the error probability in the discrimination is even lower.

\section{B. Two quantum vampires}

The capturing of two quantum vampires is more interesting. As shown in Sec. II B, we have in total three different scenarios: $\left|\psi_{\mathrm{PNR}}^{(2,0)}\right\rangle,\left|\psi_{\mathrm{PNR}}^{(1,1)}\right\rangle$, and $\left|\psi_{\mathrm{PNR}}^{(0,2)}\right\rangle$. Written in Fock state space, one has

$$
\begin{aligned}
\left|\psi_{\mathrm{PNR}}^{(2,0)}\right\rangle= & \frac{1}{\sqrt{N_{\mathrm{PNR}}^{(2,0)}}} \sum_{k=0}^{n} \sqrt{\left(\begin{array}{l}
n \\
k
\end{array}\right)}\left(\sqrt{T} T^{\prime}\right)^{n-k} \\
& \times \sqrt{(n-k)(n-k-1)}(1-T)^{\frac{k}{2}}|n-k-2, k\rangle, \\
& \left|\psi_{\mathrm{PNR}}^{(1,1)}\right\rangle=\frac{1}{\sqrt{N_{\mathrm{PNR}}^{(1,1)}}} \sum_{k=0}^{n} \sqrt{\left(\begin{array}{l}
n \\
k
\end{array}\right)} T^{\frac{n-k}{2}}
\end{aligned}
$$

$$
\begin{gathered}
\sqrt{(n-k) k}(1-T)^{\frac{k}{2}}|n-k-1, k-1\rangle, \\
\left|\psi_{\mathrm{PNR}}^{(0,2)}\right\rangle=\frac{1}{\sqrt{N_{\mathrm{PNR}}^{(0,2)}}} \sum_{k=0}^{n} \sqrt{\left(\begin{array}{l}
n \\
k
\end{array}\right)} T^{\frac{n-k}{2}} \\
\sqrt{k(k-1)}(1-T)^{\frac{k}{2}} T^{\prime k}|n-k, k-2\rangle, \\
N_{\mathrm{PNR}}^{(2,0)}=n(n-1) T^{2} T^{\prime 4}\left(1-T+T T^{\prime 2}\right)^{n-2}, \\
N_{\mathrm{PNR}}^{(1,1)}=n(n-1) T(1-T), \\
N_{\mathrm{PNR}}^{(0,2)}=n(n-1)(1-T)^{2} T^{\prime 4}\left(T+T^{\prime 2}-T T^{\prime 2}\right)^{n-2} .
\end{gathered}
$$

It should be noted that the normalized state $\left|\psi_{\mathrm{PNR}}^{(1,1)}\right\rangle$ does not contain the items with $T^{\prime}$. This is because there are two factors, $T^{\frac{n-k-1}{2}}$ and $T^{\prime \frac{k-1}{2}}$, in the coefficients of item $|n-k-1, k-1\rangle$ and they disappear after normalization. However, the transmittance of $T^{\prime}$ does have an effect on the probability of successful preparation of the state $\left|\psi_{\mathrm{PNR}}^{(1,1)}\right\rangle$.

Again, we have $M$ copies of them at hand. We will see whether the photon-number-resolving detector is sufficient for discriminating these three states. Let the mean value and variance of the state $\left|\psi_{\mathrm{PNR}}^{(2,0)}\right\rangle,\left|\psi_{\mathrm{PNR}}^{(1,1)}\right\rangle$, and $\left|\psi_{\mathrm{PNR}}^{(0,2)}\right\rangle$ be $\bar{n}_{1}, \bar{n}_{2}, \bar{n}_{3}$ and $V_{1}, V_{2}, V_{3}$, respectively. After direction calculation, it follows that

$$
\begin{gathered}
\bar{n}_{1}=\frac{(n-2) T T^{\prime 2}}{1-T+T T^{\prime 2}}, \\
\bar{n}_{2}=(n-2) T, \\
\bar{n}_{3}=\frac{(n-2) T}{T+T^{\prime 2}-T T^{\prime 2}}, \\
V_{1}=\frac{(n-2)(1-T) T T^{\prime 2}}{\left(1-T+T T^{\prime 2}\right)^{2}}, \\
V_{2}=(n-2)(1-T) T, \\
V_{3}=\frac{(n-2)(1-T) T T^{\prime 2}}{\left(T+T^{\prime 2}-T T^{\prime 2}\right)^{2}} .
\end{gathered}
$$

We use the summation of the results of photon-number detection of all $M$ measurements as an estimator $\sum_{i=1}^{M} \hat{N}_{i}$. For sufficiently large $M$, the summation will follow a Gaussian distribution:

$$
\begin{aligned}
& \sum_{i=1}^{M} \hat{N}_{i} \sim \mathcal{N}_{1}\left(M \bar{n}_{1}, M V_{1}\right), \\
& \sum_{i=1}^{M} \hat{N}_{i} \sim \mathcal{N}_{2}\left(M \bar{n}_{2}, M V_{2}\right), \\
& \sum_{i=1}^{M} \hat{N}_{i} \sim \mathcal{N}_{3}\left(M \bar{n}_{3}, M V_{3}\right),
\end{aligned}
$$

if $\left|\psi_{\mathrm{PNR}}^{(2,0)}\right\rangle^{\otimes M},\left|\psi_{\mathrm{PNR}}^{(1,1)}\right\rangle^{\otimes M},\left|\psi_{\mathrm{PNR}}^{(0,2)}\right\rangle^{\otimes M}$ are at hand.

After direct calculation, it can be easily obtained that

$$
\bar{n}_{1}<\bar{n}_{2}<\bar{n}_{3}
$$

for all $0<T, T^{\prime}<1, n>2$. A plot of the probability distribution of $\mathcal{N}_{1}, \mathcal{N}_{2}$, and $\mathcal{N}_{3}$ with increasing $M$ is given in Fig. 9. 


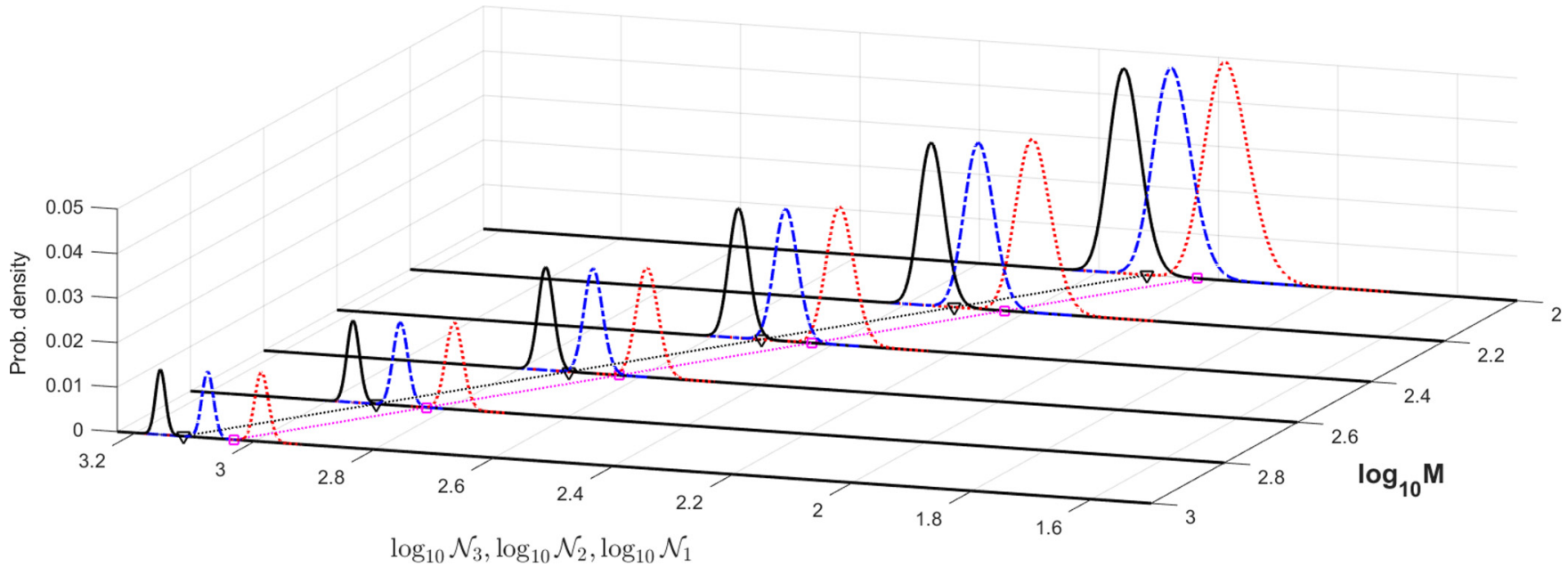

FIG. 9. Probability density of Gaussian distribution $\mathcal{N}_{1}$ (dotted red line, in logarithmic scale), $\mathcal{N}_{2}$ (dash-dotted blue line, in logarithmic scale), and $\mathcal{N}_{3}$ (solid black line, in logarithmic scale) for increasing values of $M$. Other parameters are as follows: $T=0.40, T^{\prime}=0.85, D=6$, $n=5$. Lines marked with squares indicate the value of $N_{\mathrm{th}, 1}$ of corresponding $M$. Lines marked with downward triangles indicate the $N_{\mathrm{th}, 2}$ values of the corresponding $M$.

To implement a discrimination of the three distributions, one can define two threshold values $N_{\mathrm{th}, 1}$ and $N_{\mathrm{th}, 2}$ and establish a corresponding inference rule as follows:

$$
\begin{aligned}
& A A \Leftrightarrow \rho^{(2,0) \otimes M}, \Leftrightarrow \hat{N}<N_{\text {th }, 2}, \\
& A B \Leftrightarrow \rho^{(1,1) \otimes M}, \Leftrightarrow N_{\text {th, } 1} \leqslant \hat{N}<N_{\text {th }, 2}, \\
& B B \Leftrightarrow \rho^{(0,2) \otimes M}, \Leftrightarrow \hat{N} \geqslant N_{\text {th }, 2},
\end{aligned}
$$

where we assume $N_{\text {th, } 1}<N_{\text {th }, 2}$, and we use $A A(B B)$ to denote two vampires appearing in the same $A(B)$ mode and $A B$ to denote the case in which one vampire appears in $A$ mode and the other appears in $B$ mode.

A good choice of $N_{\text {th, } 1}, N_{\text {th, } 2}$ could substantially decrease the final error probability. We define $N_{\text {th, } 1}, N_{\text {th, } 2}$ such that

$$
\begin{aligned}
& P\left(\hat{N}>N_{\text {th }, 1} \mid N_{1}\right)=P\left(\hat{N}<N_{\text {th }, 1} \mid N_{2}\right), \\
& P\left(\hat{N}>N_{\text {th }, 2} \mid N_{2}\right)=P\left(\hat{N}<N_{\text {th }, 2} \mid N_{3}\right) .
\end{aligned}
$$

Using a similar method in Sec. IV A, we can define

$$
\begin{aligned}
N_{\mathrm{th}, 1} & =\frac{M\left(\bar{n}_{2} \sqrt{V_{1}}+\bar{n}_{1} \sqrt{V_{2}}\right)}{\sqrt{V_{1}}+\sqrt{V_{2}}}, \\
N_{\mathrm{th}, 2} & =\frac{M\left(\bar{n}_{3} \sqrt{V_{2}}+\bar{n}_{2} \sqrt{V_{3}}\right)}{\sqrt{V_{2}}+\sqrt{V_{3}}} .
\end{aligned}
$$

A numerical evaluation of $N_{\text {th,1 }}$ and $N_{\text {th,2 }}$ for $T=0.40$, $T^{\prime}=0.85$ is shown in Fig. 9 .

If the three configurations $(2,0),(1,1)$, and $(0,2)$ appear with equal probability, the error probability in the presence of two quantum vampires can be given by

$$
\begin{aligned}
P_{\mathrm{err}}^{\prime}= & \frac{1}{3} P\left(\hat{N}>N_{\mathrm{th}, 1} \mid \mathcal{N}_{1}\right) \\
& +\frac{1}{3}\left[P\left(\hat{N}<N_{\mathrm{th}, 1} \mid \mathcal{N}_{2}\right)+P\left(\hat{N}>N_{\mathrm{th}, 2} \mid \mathcal{N}_{2}\right)\right] \\
& +\frac{1}{3} P\left(\hat{N}<N_{\mathrm{th}, 2} \mid \mathcal{N}_{3}\right)
\end{aligned}
$$

$$
\begin{aligned}
= & \frac{1}{3} \operatorname{erfc}\left(\sqrt{\frac{M}{2}} \frac{\bar{n}_{2}-\bar{n}_{1}}{\sqrt{V_{2}}+\sqrt{V_{1}}}\right) \\
& +\frac{1}{3} \operatorname{erfc}\left(\sqrt{\frac{M}{2}} \frac{\bar{n}_{3}-\bar{n}_{2}}{\sqrt{V_{2}}+\sqrt{V_{3}}}\right) \\
\approx & \frac{e^{-M R_{\mathrm{eff}, 1}}}{3 \sqrt{\pi M R_{\mathrm{eff}, 1}}}+\frac{e^{-M R_{\mathrm{eff}, 2}}}{3 \sqrt{\pi M R_{\mathrm{eff}, 2}}},
\end{aligned}
$$

with

$$
R_{\mathrm{eff}, 1}=\frac{\left(\bar{n}_{2}-\bar{n}_{1}\right)^{2}}{2\left(\sqrt{V_{2}}+\sqrt{V_{1}}\right)^{2}}, \quad R_{\mathrm{eff}, 2}=\frac{\left(\bar{n}_{3}-\bar{n}_{2}\right)^{2}}{2\left(\sqrt{V_{3}}+\sqrt{V_{2}}\right)^{2}}
$$

As an explicit example, we show the error probability of identifying two quantum vampires in the case of $T=$ $0.40, T^{\prime}=0.85$. In Fig. 10, we plot $P_{\text {err }}^{\prime}$ logarithmically as a function of copy number $M$. It is evident that a steady decrease in error probability is observed. In particular, for $M=10^{2}=$ $100, P_{\mathrm{err}}^{\prime}=0.0555$. This means that two realistic quantum vampires can also be identified using the photon-numberresolving detector. In Fig. 10, we also give a plot of the quantum Chernoff bound $P_{\mathrm{QCB}}^{(20,11)}$ of discriminating $\left\{\left|\psi_{\mathrm{PNR}}^{(2,0)}\right\rangle\right.$, $\left.\left|\psi_{\mathrm{PNR}}^{(1,1)}\right\rangle\right\}, P_{\mathrm{QCB}}^{(11,02)}$ of discriminating $\left\{\left|\psi_{\mathrm{PNR}}^{(1,1)}\right\rangle,\left|\psi_{\mathrm{PNR}}^{(0,2)}\right\rangle\right\}$, and $P_{\mathrm{QCB}}^{(20,02)}$ of discriminating $\left\{\left|\psi_{\mathrm{PNR}}^{(2,0)}\right\rangle,\left|\psi_{\mathrm{PNR}}^{(0,2)}\right\rangle\right\}$, if joint measurement is performed on $M$ copy quantum states. Although there is still a gap between our mode-by-mode scheme and the ideal bounds of $P_{\mathrm{OCB}}^{(20,11)}, P_{\mathrm{QCB}}^{(11,02)}, P_{\mathrm{QCB}}^{(20,02)}$, our work shows that an exponential decrease in error probability can still be achieved.

In a brief summary, we give examples of capturing one and two quantum vampires using mode-by-mode photon-number measurement. Our method can be generalized straightforwardly to capture more and more quantum vampires. 


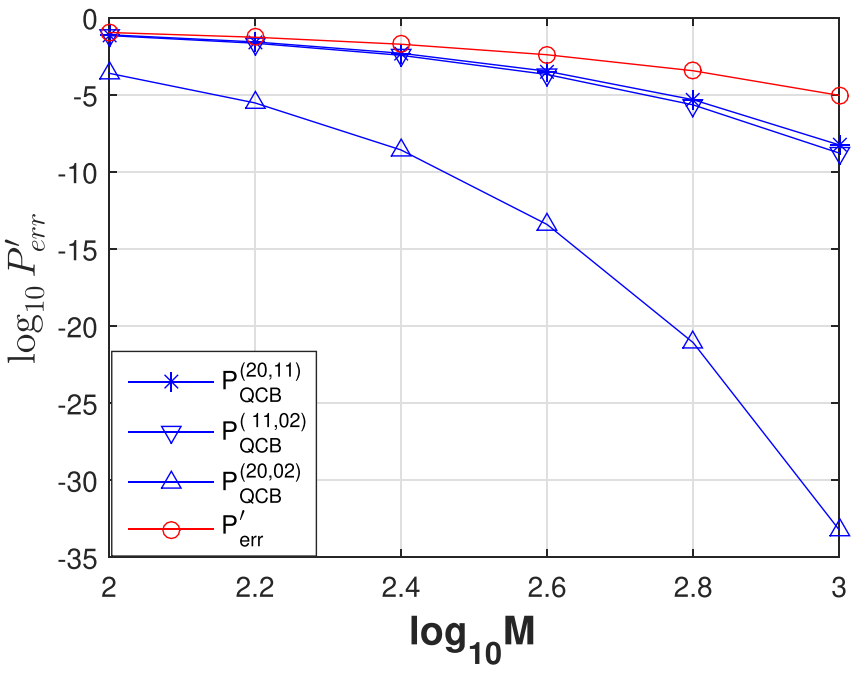

FIG. 10. $P_{\text {err }}^{\prime}$ and quantum Chernoff bound (both in logarithmic scale) of discriminating two state of $\left\{\left|\psi_{\mathrm{PNR}}^{(2,0)}\right\rangle,\left|\psi_{\mathrm{PNR}}^{(1,1)}\right\rangle\right\},\left\{\left|\psi_{\mathrm{PNR}}^{(1,1)}\right\rangle\right.$, $\left.\left|\psi_{\mathrm{PNR}}^{(0,2)}\right\rangle\right\}$, and $\left\{\left|\psi_{\mathrm{PNR}}^{(0,2)}\right\rangle,\left|\psi_{\mathrm{PNR}}^{(0,2)}\right\rangle\right\}$. All other parameters are the same as Fig. 9.

\section{CONCLUSIONS}

The quantum vampire effect is an interesting phenomenon in quantum optics. It originates from an interesting property of the annihilation operator $\hat{a}|0\rangle=0$ [as frequently used in Eqs. (2) and (3)]. Original research shows that applying a single annihilation operation on a single mode leaves no shadows. Our work further shows that applying annihilation to different optical modes and applying annihilation multiple times leaves no shadow either. Furthermore, we show that ideal annihilation, if implemented in realistic scenarios with beam splitters, will definitely leave some shadows. It is possible to construct optimal quantum measurement schemes for state discrimination to identify the location where the annihilation operation is applied. Moreover, two-time photon annihilation on the same mode and one-time annihilation on each mode also leave different shadows, which means that ideal quantum vampires may be illuminated or identified in real scenarios. Such a result sheds more light on quantum vampires and offers a theoretical guidance to develop a more powerful quantum vampire.

\section{ACKNOWLEDGMENTS}

This work is supported by National Natural Science Foundation of China (NSFC) (Grants No. 11574400 and No. 11204379), Beijing Institute of Technology Research Fund Program for Young Scholars, and the joint program of ICTP (Trieste, Italy)-NSFC (Grant No. 11981240356).

\section{APPENDIX A: DERIVATION OF THE OUTPUT STATE OF REALISTIC PHOTON SUBTRACTION WITH ON-OFF PHOTON DETECTORS}

\section{Photon substraction on mode $A$}

The initial state of modes $A, B, C$ are $|n\rangle,|0\rangle,|0\rangle$ and the coupling before $A, B$, and $C$ (before photon detection) follows:

$$
\left|\psi_{A B C}^{(A)}\right\rangle=U_{A C}\left(T^{\prime}\right) U_{A_{0} B_{0}}(T)|n\rangle_{A_{0}}|0\rangle_{B}|0\rangle_{C} .
$$

Through a successful distillation event, mode $C$ is projected onto nonvacuum components and the state of modes $A$ and $B$ is reduced to

$$
\rho^{(1,0)}=\frac{1}{P_{C}^{(1,0)}} \operatorname{Tr}_{C}\left[\left|\psi_{A B C}^{(A)}\right\rangle\left\langle\psi_{A B C}^{(A)}\right|\left(I_{A B} \otimes \hat{\Pi}_{C}^{\mathrm{on}}\right)\right],
$$

with $P_{C}^{(1,0)}=\operatorname{Tr}\left[\left|\psi_{A B C}^{(A)}\right\rangle\left\langle\psi_{A B C}^{(A)}\right| I_{A B} \otimes \hat{\Pi}_{C}^{\text {on }}\right], \hat{\Pi}_{C}^{\text {on }}=I_{\infty}-|0\rangle\langle 0|=$ $\sum_{m=1}^{\infty}|m\rangle\langle m|$ and $\operatorname{Tr}_{C}$ denotes the partial trace of the transmittance over the optical mode $C$. After direct calculation, we obtain the following:

$$
\begin{aligned}
\rho^{(1,0)}= & \frac{\left(T T^{\prime}\right)^{n}}{1-\left(1-T+T T^{\prime}\right)^{n}} \\
& \times \sum_{k=0}^{n-1} \sum_{k^{\prime}=0}^{n-1} \sum_{l=1}^{\min \left(n-k, n-k^{\prime}\right)} \sqrt{\left(\begin{array}{l}
n \\
k
\end{array}\right)\left(\begin{array}{l}
n \\
k^{\prime}
\end{array}\right)\left(\begin{array}{c}
n-k \\
l
\end{array}\right)\left(\begin{array}{c}
n-k^{\prime} \\
l
\end{array}\right)} \\
& \times\left(\frac{1-T}{T T^{\prime}}\right)^{\frac{k+k^{\prime}}{2}}\left(\frac{1-T^{\prime}}{T^{\prime}}\right)^{l}|n-k-l\rangle_{A} \\
& \times\left\langle n-k^{\prime}-l|\otimes| k\right\rangle_{B}\left\langle k^{\prime}\right| .
\end{aligned}
$$

\section{Photon substraction on mode $B$}

Similar analysis can be performed on the subtraction of mode $B$. We obtain

$$
\left|\psi_{A B C}^{(B)}\right\rangle=U_{B C}\left(T^{\prime}\right) U_{A_{0} B_{0}}(T)|n\rangle_{A_{0}}|0\rangle_{B_{0}}|0\rangle_{C}
$$

and

$$
\begin{aligned}
\rho^{(0,1)}= & \operatorname{Tr}_{C}\left[\left|\psi_{A B C}^{(B)}\right\rangle\left\langle\psi_{A B C}^{(B)}\right|\left(I_{A B} \otimes \hat{\Pi}_{C}^{\text {on }}\right)\right] \\
= & \frac{T^{n}}{1-\left(T+T^{\prime}-T T^{\prime}\right)^{n}} \\
& \times \sum_{k=1}^{n} \sum_{k^{\prime}=1}^{n} \sum_{l=1}^{\min \left(k, k^{\prime}\right)} \sqrt{\left(\begin{array}{l}
n \\
k
\end{array}\right)\left(\begin{array}{l}
n \\
k^{\prime}
\end{array}\right)\left(\begin{array}{l}
k \\
l
\end{array}\right)\left(\begin{array}{l}
k^{\prime} \\
l
\end{array}\right)} \\
& \times\left(\frac{(1-T) T^{\prime}}{T}\right)^{\frac{k+k^{\prime}}{2}}\left(\frac{1-T^{\prime}}{T^{\prime}}\right)^{l}|n-k\rangle_{A} \\
& \times\left\langle n-k^{\prime}|\otimes| k-l\right\rangle_{B}\left\langle k^{\prime}-l\right| .
\end{aligned}
$$

\section{APPENDIX B: FIDELITY OF ONE-TIME PHOTON ANNIHILATION WITH ON-OFF PHOTON DETECTOR}

It is interesting to evaluate the fidelity of ideal one-time photon subtraction. For convenience, we consider single-side photon subtraction on the $A$ mode. Ideal photon subtraction will generate the pure state $\left|\widetilde{\psi}_{A B}^{(1,0)}\right\rangle$ in Eq. (2). After normalization, one obtains

$$
\begin{aligned}
\left|\psi_{A B}^{(1,0)}\right\rangle= & \frac{\left|\widetilde{\psi}_{A B}^{(1,0)}\right\rangle}{\sqrt{\left\langle\widetilde{\psi}_{A B}^{(1,0)} \mid \widetilde{\psi}_{A B}^{(1,0)}\right\rangle}} \\
= & \frac{1}{\sqrt{n(n-1)}} \sum_{k=0}^{n-1} \sqrt{\left(\begin{array}{l}
n \\
k
\end{array}\right)(T)^{\frac{n-k}{2}-1}(1-T)^{\frac{k}{2}}} \\
& \times \sqrt{n-k}|n-k-1\rangle|k\rangle .
\end{aligned}
$$


However, if one uses the realistic photon subtraction in Fig. 3(a), the output state is $\rho^{(1,0)}$, as given in Eq. (A3). The fidelity can be defined by

$$
\begin{aligned}
F & =\left\langle\psi_{A B}^{(1,0)}\left|\rho^{(1,0)}\right| \psi_{A B}^{(1,0)}\right\rangle \\
& =\frac{T^{\prime n-1} T^{n-2}\left(1-T^{\prime}\right)}{\left[1-\left(1-T+T T^{\prime}\right)^{n}\right] n(n-1)} \mathcal{W}^{2},
\end{aligned}
$$

with

$$
\begin{aligned}
\mathcal{W} & =\sum_{k=0}^{n}\left(\begin{array}{l}
n \\
k
\end{array}\right) T^{\frac{n-k}{2}}\left(\frac{(1-T)^{2}}{T T^{\prime}}\right)^{\frac{k}{2}} \sqrt{n-k} \\
& <\sum_{k=0}^{n}\left(\begin{array}{l}
n \\
k
\end{array}\right) T^{\frac{n-k}{2}}\left(\frac{(1-T)^{2}}{T T^{\prime}}\right)^{\frac{k}{2}} \sqrt{n} \\
& =\sqrt{n} T^{\frac{n}{2}}\left(1+\frac{1}{T \sqrt{T^{\prime}}}-\frac{1}{\sqrt{T^{\prime}}}\right)^{n} .
\end{aligned}
$$

Finally, we obtain

$$
\begin{aligned}
F<F^{U}= & \frac{T^{n-1} T^{2 n-2}\left(1-T^{\prime}\right)}{\left[1-\left(1-T+T T^{\prime}\right)^{n}\right](n-1)} \\
& \times\left(1+\frac{1}{T \sqrt{T^{\prime}}}-\frac{1}{\sqrt{T^{\prime}}}\right)^{2 n} .
\end{aligned}
$$

Note that $T^{\prime}$ is the transmittance of the beam splitter used for photon subtraction. When considering photon subtraction, we are mainly interested in the case when $T^{\prime}>0.9$, or $1-T^{\prime} \ll$ 1 . The upper bound of fidelity $F^{U}$ is then given by

$$
\begin{aligned}
F^{U}= & \frac{1}{n(n-1) T^{3}}-\frac{(n T+T-2)}{2 n(n-1) T^{3}}\left(1-T^{\prime}\right) \\
& +\frac{n^{2} T^{2}+3 n T^{2}-T^{2}-9 n T-6 T+12}{12 n(n-1) T^{3}}\left(1-T^{\prime}\right)^{2} \\
& +O\left(1-T^{\prime}\right)^{3} .
\end{aligned}
$$

Therefore, the scheme in Fig. 3 performs better if one uses a beam splitter with higher transmittance $T^{\prime}$.
[1] C. H. Bennett and G. Brassard, in Proceedings of the IEEE International Conference on Computers, Systems, and Signal Processing, Bangalore, India (IEEE, New York, 1984), p. 175.

[2] V. Giovannetti, S. Lloyd, and L. Maccone, Quantum-enhanced measurements: Beating the standard quantum limit, Science 306, 1330 (2004).

[3] C. H. Bennett, G. Brassard, C. Crepeau, R. Jozsa, A. Peres, and W. Wootters, Teleporting an Unknown Quantum State via Dual Classical and EPR Channels, Phys. Rev. Lett. 70, 1895 (1993).

[4] P. W. Shor, Algorithms for quantum computation: Discrete log and factoring, in Proceedings of the 35th Annual Symposium on the Foundations of Computer Science, edited by S. Goldwasser (IEEE Computer Society Press, Los Alamitos, CA, 1994), p. 124.

[5] I. A. Fedovro, A. E. Ulanov, Y. V. Kurochkin, and A. I. Lvovsky, Quantum vampire: Collapse-free action at a distance by the photon annihilation operator, Optica 2, 112 (2015).

[6] K. G. Katamadze, G. V. Avosopiants, Y. I. Bogdanov, and S. P. Kulik, How quantum is the "quantum vampire" effect?: Testing with thermal light, Optica 5, 723 (2018).

[7] K. G. Katamadze, E. V. Kovlakov, G. V. Avosopiants, and S. P. Kulik, Direct test of the "quantum vampire's" shadow absence with use of thermal light, Opt. Lett. 44, 3286 (2019).

[8] N. Lütkenhaus, Security against individual attacks for realistic quantum key distribution, Phys. Rev. A 61, 052304 (2000).

[9] X. B. Wang, Beating the Photon-Number-Splitting Attack in Practical Quantum Cryptography, Phys. Rev. Lett. 94, 230503 (2005).

[10] H.-K. Lo, X. Ma, and K. Chen, Decoy State Quantum Key Distribution, Phys. Rev. Lett. 94, 230504 (2005).

[11] M. O. Scully and M. S. Zubairy, Quantum Optics (Cambridge University Press, Cambridge, UK, 1996).

[12] T. Opatrný, G. Kurizki, and D. G. Welsch, Improvement on teleportation of continuous variables by photon subtraction via conditional measurement, Phys. Rev. A 61, 032302 (2000).
[13] S. Olivares, M. G. A. Paris, and R. Bonifacio, Teleportation improvement by inconclusive photon subtraction, Phys. Rev. A 67, 032314 (2003).

[14] A. Kitagawa, M. Takeoka, M. Sasaki, and A. Chefles, Entanglement evaluation of non-Gaussian states generated by photon subtraction from squeezed states, Phys. Rev. A 73, 042310 (2006).

[15] M. Sasaki and S. Suzuki, Multimode theory of measurementinduced non-Gaussian operation on wideband squeezed light: Analytical formula, Phys. Rev. A 73, 043807 (2006).

[16] S. L. Zhang and P. van Loock, Distillation of mixedstate continuous-variable entanglement by photon subtraction, Phys. Rev. A 82, 062316 (2010).

[17] A. P. Lund and T. C. Ralph, Continuous-variable entanglement distillation over a general lossy channel, Phys. Rev. A 80, 032309 (2009).

[18] S. L. Zhang, J. H. Shi, C. H. Jin, X. B. Zou, B. S. Shi, and G. C. Guo, Distillation of phase-damped continuous-variable entanglement with photon subtraction, J. Opt. Soc. Am. B 30, 1922 (2013).

[19] H. Takahashi, J. S. Neergaard-Nielsen, M. Takeuchi, M. Takeoka, K. Hayasaka, A. Furusawa, and M. Sasaki, Entanglement distillation from Gaussian input states, Nat. Photon. 4, 178 (2010).

[20] R. García-Patrón, J. Fiurášek, N. J. Cerf, J. Wenger, R. TualleBrouri, and P. Grangier, Proposal for a Loophole-Free Bell Test Using Homodyne Detection, Phys. Rev. Lett. 93, 130409 (2004).

[21] H. Nha and H. J. Carmichael, Proposed Test of Quantum Nonlocality for Continuous Variables, Phys. Rev. Lett. 93, 020401 (2004).

[22] S. Zhang and X. Zhang, Distillation of single-mode-squeezed entangled state in presence of strong squeezing, OSA Continuum 2, 2050 (2019).

[23] M. F. Sacchi, Optimal discrimination of quantum operations, Phys. Rev. A 71, 062340 (2005). 
[24] M. F. Sacchi, Entanglement can enhance the distinguishability of entanglement-breaking channels, Phys. Rev. A 72, 014305 (2005).

[25] C. W. Helstrom, Quantum detection and estimation theory, J. Stat. Phys. 1, 231 (1969).

[26] K. M. R. Audenaert, J. Calsamiglia, R. Munoz-Tapia, E. Bagan, L. Masanes, A. Acin, and F. Verstraete, Discriminating States: The Quantum Chernoff Bound, Phys. Rev. Lett. 98, 160501 (2007).

[27] J. Calsamiglia, R. Muñoz-Tapia, L. Masanes, A. Acin, and E. Bagan, Quantum Chernoff bound as a measure of distinguishability between density matrices: Application to qubit and Gaussian states, Phys. Rev. A 77, 032311 (2008).

[28] N. Namekata, Y. Takahashi, G. Fujii, D. Fukuda, S. Kurimura, and S. Inoue, Non-Gaussian operation based on photon subtraction using a photon-number-resolving detector at a telecommunications wavelength, Nat. Photon. 4, 655 (2010).

[29] F. Mattioli, Z. Zhou, A. Gaggero, R. Gaudio, S. Jahanmirinejad, D. Sahin, F. Marsili, R. Leoni, and A. Fiore, Photon-numberresolving superconducting nanowire detectors, Supercond. Sci. Technol. 28, 104001 (2015). 